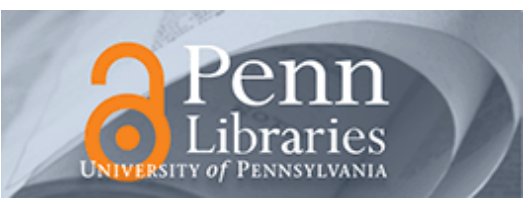

University of Pennsylvania

ScholarlyCommons

9-4-2012

\title{
Direct Determination of the Effect of Strain on Domain Morphology in Ferroelectric Superlattices with Scanning Probe Microscopy
}

\author{
K. Kathan-Galipeau \\ University of Pennsylvania \\ P. P. Wu \\ Pennsylvania State University
}

Y. L. Li

Pacific Northwest National Laboratory

L. Q. Chen

Pennsylvania State University

A Soukiassian

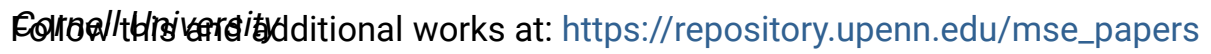

Part of the Materials Science and Engineering Commons

See next page for additional authors

\section{Recommended Citation}

Kathan-Galipeau, K., Wu, P. P., Li, Y. L., Chen, L. Q., Soukiassian, A., Zhu, Y., Muller, D. A., Xi, X. X., Schlom, D. G., \& Bonnell, D. A. (2012). Direct Determination of the Effect of Strain on Domain Morphology in Ferroelectric Superlattices with Scanning Probe Microscopy. Retrieved from https://repository.upenn.edu/ mse_papers/217

\footnotetext{
K. Kathan-Galipeau, P. P. Wu, Y. L. Li, L. Q. Chen, A. Soukiassian, Y. Zhu, D. A. Muller, X. X. Xi, D. G. Schlom, and D. A. Bonnell. (2012). Direct determination of the effect of strain on domain morphology in ferroelectric superlattices with scanning probe microscopy. Journal of Applied Physics, 112(5), 052011. doi: 10.1063/1.4746081 Copyright 2012 American Institute of Physics. This article may be downloaded for personal use only. Any other use requires prior permission of the author and the American Institute of Physics.
}

This paper is posted at ScholarlyCommons. https://repository.upenn.edu/mse_papers/217

For more information, please contact repository@pobox.upenn.edu. 


\title{
Direct Determination of the Effect of Strain on Domain Morphology in Ferroelectric Superlattices with Scanning Probe Microscopy
}

\author{
Abstract \\ A variant of piezo force microscopy was used to characterize the effect of strain on polarization in \\ $\left[\left(\mathrm{BaTiO}_{3}\right)_{n} /\left(\mathrm{SrTiO}_{3}\right)_{m}\right]_{p}$ superlattices. The measurements were compared to theoretical predictions based \\ on phase-field calculations. When polarization is constrained to be perpendicular to the substrate, the \\ measured polarization and domain morphology agree quantitatively with the predictions. This case allows \\ the presence of an internal electric field in the thin film to be identified. The measured trend in \\ piezoelectric response with strain state was in qualitative agreement with predictions, and the differences \\ were consistent with the presence of internal electrical fields. Clear differences in domain morphology \\ with strain were observed; and in some cases, the lateral anisotropic strain appeared to influence the \\ domain morphology. The differences in magnitude and morphology were attributed to the internal electric \\ fields and anisotropic strains. \\ Disciplines \\ Materials Science and Engineering

\section{Comments} \\ K. Kathan-Galipeau, P. P. Wu, Y. L. Li, L. Q. Chen, A. Soukiassian, Y. Zhu, D. A. Muller, X. X. Xi, D. G. Schlom, \\ and D. A. Bonnell. (2012). Direct determination of the effect of strain on domain morphology in \\ ferroelectric superlattices with scanning probe microscopy. Journal of Applied Physics, 112(5), 052011. \\ doi: $10.1063 / 1.4746081$ \\ Copyright 2012 American Institute of Physics. This article may be downloaded for personal use only. Any \\ other use requires prior permission of the author and the American Institute of Physics.

\section{Author(s)} \\ K. Kathan-Galipeau, P. P. Wu, Y. L. Li, L. Q. Chen, A Soukiassian, Y. Zhu, D. A. Muller, X. X. Xi, D. G. Schlom, \\ and Dawn A. Bonnell
}




\title{
Direct determination of the effect of strain on domain morphology in ferroelectric superlattices with scanning probe microscopy
}

\author{
K. Kathan-Galipeau, ${ }^{1}$ P. P. Wu, ${ }^{2}$ Y. L. Li, ${ }^{3}$ L. Q. Chen, ${ }^{2,4}$ A. Soukiassian, ${ }^{5}$ Y. Zhu, ${ }^{6}$ \\ D. A. Muller, ${ }^{6,7}$ X. X. Xi, ${ }^{2, a)}$ D. G. Schlom, ${ }^{5,7}$ and D. A. Bonnell ${ }^{1}$ \\ ${ }^{1}$ Department of Materials Science, The University of Pennsylvania, Philadelphia, Pennsylvania 19104, USA \\ ${ }^{2}$ Materials Research Institute, The Pennsylvania State University, University Park, Pennsylvania 16802, USA \\ ${ }^{3}$ Pacific Northwest National Laboratory, Richland, Washington 99352, USA \\ ${ }^{4}$ Materials Science Department, The Pennsylvania State University, University Park, Pennsylvania 16802, USA \\ ${ }^{5}$ Department of Materials Science and Engineering, Cornell University, Ithaca, New York 14853, USA \\ ${ }^{6}$ School of Applied and Engineering Physics, Cornell University, Ithaca, New York 14853, USA \\ ${ }^{7}$ Kavli Institute at Cornell for Nanoscale Science, Ithaca, New York 14853, USA
}

(Received 27 December 2011; accepted 11 July 2012; published online 4 September 2012)

\begin{abstract}
A variant of piezo force microscopy was used to characterize the effect of strain on polarization in $\left[\left(\mathrm{BaTiO}_{3}\right)_{n} /\left(\mathrm{SrTiO}_{3}\right)_{m}\right]_{p}$ superlattices. The measurements were compared to theoretical predictions based on phase-field calculations. When polarization is constrained to be perpendicular to the substrate, the measured polarization and domain morphology agree quantitatively with the predictions. This case allows the presence of an internal electric field in the thin film to be identified. The measured trend in piezoelectric response with strain state was in qualitative agreement with predictions, and the differences were consistent with the presence of internal electrical fields. Clear differences in domain morphology with strain were observed; and in some cases, the lateral anisotropic strain appeared to influence the domain morphology. The differences in magnitude and morphology were attributed to the internal electric fields and anisotropic strains. (C) 2012 American Institute of Physics. [http://dx.doi.org/10.1063/1.4746081]
\end{abstract}

\section{INTRODUCTION}

Advances in epitaxial oxide thin film fabrication, in combination with developments in the theory of complex oxide behavior, have inspired a new class of ferroelectric materials based on the control of lattice strain. ${ }^{1-8}$ In particular, a new family of materials in which ferroelectric and dielectric constituents are combined in epitaxial superlattices with atomic layer thickness precision has recently been developed. Thin films can be commensurately strained to percent levels without fracture via lattice mismatch strain to an underlying substrate, $^{7}$ enabling enhancement of ferroelectric transition temperatures by hundreds of degrees, ${ }^{7,25}$ the transformation of materials that are normally never ferroelectric into ferroelectrics, ${ }^{9-12}$ or totally new phenomena to emerge. ${ }^{13-15}$

Essential to understanding the fundamental relation between strain and polarization in superlattices is the analysis of the associated domain morphology. The domain morphology is a direct consequence of energy minimization in the system. For example in homogeneous single component films, the case of $180^{\circ}$ stripe domains has been considered in detail. $^{16-18}$ These in-plane structures minimize the total free energy including contributions from the domain walls and the depolarization fields. ${ }^{19}$ The roles of crystal symmetry, film thickness, electrostatic boundary conditions (surface and interface charge compensation), and temperature have been investigated in an effort to understand the ferroelectric ground state and to achieve the desired mono domain structure.

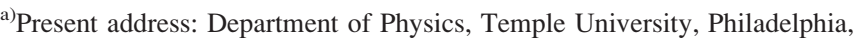
Pennsylvania 19122, USA.
}

While superlattices of ferroelectric and non-ferroelectric compounds can be designed to induce novel properties, ${ }^{20-25}$ the polarization is more complex than that of single component films. The magnitude and orientation of polarization in the non-ferroelectric layers is unknown, but manifests in the domain morphology. The relation between strain and polarization in ferroelectric thin films is determined from macroscopic measurements, such as $\mathrm{x}$-ray diffraction, optical second harmonic generation, and more recently Raman spectroscopy. ${ }^{26,27}$ In these cases, the lateral morphologies of the domains are not directly determined. Recently, Streiffer et al. have used electrostatic force imaging (or surface potential imaging) of epitaxial $\mathrm{PbTiO}_{3}$ thin films to map changes in domain morphology, providing a visualization of domains. ${ }^{18}$ This approach is effective in characterizing domain morphology and in some cases domain dynamics, but does not yield properties, i.e., polarization.

Advances in scanning probe microscopy techniques allow an increasing range of properties to be measured at local scales. Piezo force microscopy (PFM) can, in principle, determine not only the local polarization orientation but also the magnitude of the polarization, though in the general case, quantification is complex. Nevertheless, it has been used semi-quantitatively in studies of ferroelectric domain morphology and domain dynamics. ${ }^{28}$ Until recently, direct imaging of domain morphology in superlattices was not possible due to the combination of small domain size and small piezoelectric response. KathanGalipeau et al. ${ }^{29}$ recently demonstrated an experimental approach that enables quantitative characterization of this class of materials by combining dual-frequency PFM with pointwise normalization of local contact properties. 


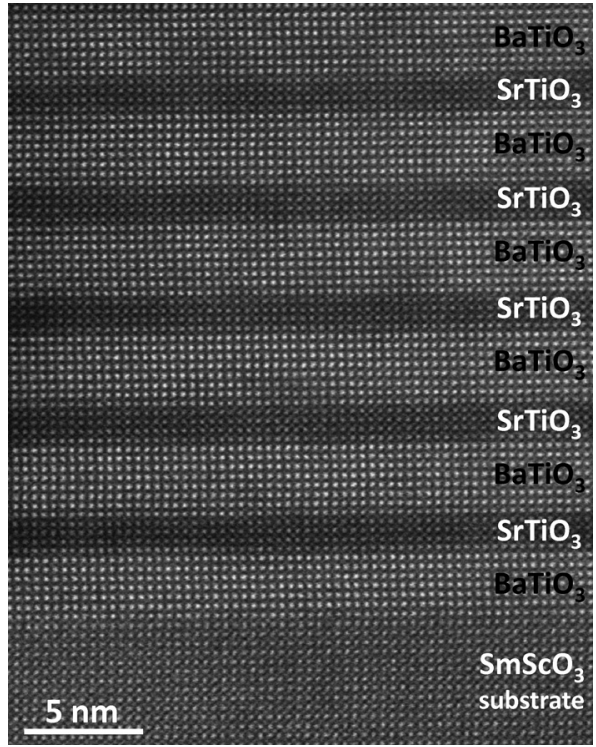

FIG. 1. Cross-sectional scanning transmission electron microscope image showing the structure of the $\left[\left(\mathrm{BaTiO}_{3}\right)_{n} /\left(\mathrm{SrTiO}_{3}\right)_{m}\right]_{p}$ superlattices studied. The particular superlattice shown is the $\left[\left(\mathrm{BaTiO}_{3}\right)_{8} /\left(\mathrm{SrTiO}_{3}\right)_{4}\right]_{40}$ superlattice grown on (101) $\mathrm{SmScO}_{3}$. The other two superlattices studied were $\left[\left(\mathrm{BaTiO}_{3}\right)_{8} /\right.$ $\left.\left(\mathrm{SrTiO}_{3}\right)_{4}\right]_{40}$ on $(101) \mathrm{GdScO}_{3}$ and $\left[\left(\mathrm{BaTiO}_{3}\right)_{3} /\left(\mathrm{SrTiO}_{3}\right)_{4}\right]_{25}$ on $(001) \mathrm{SrTiO}_{3}$.

Here, we use the widely studied model system of ferroelectric superlattices $\left[\left(\mathrm{BaTiO}_{3}\right)_{n} /\left(\mathrm{SrTiO}_{3}\right)_{m}\right]_{p}$, where $n$ and $m$ refer to the thickness, in psueducubic (001)-oriented unit cells of the $\mathrm{BaTiO}_{3}$ and $\mathrm{SrTiO}_{3}$ layers and $p$ to the number of times these layers are repeated, as a platform to systematically examine the effect of strain on polarization. A systematic variation in strain is induced by depositing such epitaxial superlattices on substrates with various degrees of lattice mismatch. Heterostructure design enables systematic variation of boundary conditions and recently developed phase-field calculations provide a theoretical framework to relate experimental observations to polarization.

\section{EXPERIMENTAL AND THEORETICAL PROCEDURES}

Figure 1 illustrates one of the $\left[\left(\mathrm{BaTiO}_{3}\right)_{n} /\left(\mathrm{SrTiO}_{3}\right)_{m}\right]_{p}$ superlattice heterostructures studied, specifically $\left[\left(\mathrm{BaTiO}_{3}\right)_{8} /\right.$ $\left.\left(\mathrm{SrTiO}_{3}\right)_{4}\right]_{40}$ grown on a (101) $\mathrm{SmScO}_{3}$ substrate. [In this manuscript, we use the standard setting of space group \#62, Pnma, to describe the crystallography of $\mathrm{SmScO}_{3}$ and
$\mathrm{GdScO}_{3}$. Although some authors use this setting, many others use the non-standard setting $\mathrm{Pbnm}$ for $\mathrm{SmScO}_{3}$ and other perovskites with the $\mathrm{GdFeO}_{3}$ crystal structure, where the orientation of our substrates would be described as (110) $\mathrm{SmScO}_{3}$ and (110) $\mathrm{GdScO}_{3}$.] The other two superlattices investigated were $\left[\left(\mathrm{BaTiO}_{3}\right)_{8} /\left(\mathrm{SrTiO}_{3}\right)_{4}\right]_{40}$ grown on (101) $\mathrm{GdScO}_{3}$ and $\left[\left(\mathrm{BaTiO}_{3}\right)_{3} /\left(\mathrm{SrTiO}_{3}\right)_{4}\right]_{25}$ grown on $\mathrm{TiO}_{2}$-terminated ${ }^{30}$ (001) $\mathrm{SrTiO}_{3}$. Film thicknesses are $192 \mathrm{~nm}, 192 \mathrm{~nm}$, and $70 \mathrm{~nm}$, respectively. The $\left[\left(\mathrm{BaTiO}_{3}\right)_{n} /\left(\mathrm{SrTiO}_{3}\right)_{m}\right]_{p}$ superlattices were fabricated by molecular-beam epitaxy (MBE) by sequential shuttered deposition of precise singlemonolayer doses of $\mathrm{BaO}, \mathrm{SrO}$, and $\mathrm{TiO}_{2}$ at a substrate temperature of $\sim 650{ }^{\circ} \mathrm{C}$ in a background pressure of $5 \times 10^{-7}$ Torr of molecular oxygen. ${ }^{31}$ Four-circle $\mathrm{x}$-ray diffraction analysis confirmed that all three $\left[\left(\mathrm{BaTiO}_{3}\right)_{n} /\right.$ $\left.\left(\mathrm{SrTiO}_{3}\right)_{m}\right]_{p}$ superlattice samples were commensurate with the underlying substrates. ${ }^{30}$ The lattice mismatches in these samples are summarized in Table I.

The domain morphology was characterized by PFM, which operates by locating a conducting probe tip on a surface, applying an ac electric field and measuring the resulting expansion/contraction due to the inverse piezoelectric effect. The expansion/contraction is quantified by detecting the motion of the cantilever to which an Au-coated tip is attached at a frequency near the resonance of the cantilever in contact with the surface. Images were acquired with tip-free resonance $=79.14 \mathrm{kHz}$, cantilever spring constant $k=2.23 \mathrm{~N} / \mathrm{m}$, oscillation amplitude $=6.6 \mathrm{~V}$, and contact force $\sim 60 \mathrm{nN}$. Several factors can affect the interpretation of the PFM signal in terms of properties. The contact resonance is known to vary with position on a surface, an effect that is usually ignored. In dual frequency PFM, a frequency above and one below the resonance are excited. ${ }^{32}$ Both amplitudes are detected and used to shift frequencies, so that contact resonance variations are eliminated from the data. In addition, the contribution of the local contact resonance to the amplitude of the response can be calculated and subtracted to determine the absolute value of the property. ${ }^{29,33}$ Non-local electrostatic fields from the tip shaft and cantilever can contribute a spurious force component leading to artifacts. This effect can be minimized by imaging with a sufficiently large contact force. ${ }^{34,35}$ We have shown how this can lead to quantification of polarization in cases where the sample configuration and crystallographic orientations are controlled. $^{29}$

TABLE I. Comparison of substrate and film lattice dimension differences.

\begin{tabular}{|c|c|c|c|c|}
\hline Substrate & $\begin{array}{c}\mathrm{SrTiO}_{3} \\
\text { unstrained } a=b=0.3905 \mathrm{~nm}\end{array}$ & $\begin{array}{c}\mathrm{BaTiO}_{3} \\
\text { unstrained } a=b=0.4005 \mathrm{~nm}\end{array}$ & $\begin{array}{l}\text { Calc. average vertical } \\
\text { polarization in } \mathrm{cm}^{-2}\end{array}$ & $\begin{array}{c}\text { Measured } \\
\text { piezo response }\end{array}$ \\
\hline $\begin{array}{l}\mathrm{SrTiO}_{3} \\
a=b=0.3905 \mathrm{~nm}\end{array}$ & $0 \mathrm{~nm}(0 \%)$ & $-0.010 \mathrm{~nm}(-2.5 \%)$ & 0.103 & $0.8 \mathrm{pm} / \mathrm{V}$ \\
\hline \multicolumn{5}{|l|}{$\mathrm{GdScO}_{3}$} \\
\hline$a=0.3966 \mathrm{~nm}$ & $0.0061 \mathrm{~nm}(1.6 \%)$ & $-0.0039 \mathrm{~nm}(-1.0 \%)$ & 0.0470 & $0.6 \mathrm{pm} / \mathrm{V}$ \\
\hline$b=0.3970 \mathrm{~nm}$ & $0.0065 \mathrm{~nm}(1.7 \%)$ & $-0.0035 \mathrm{~nm}(-0.9 \%)$ & - & - \\
\hline \multicolumn{5}{|l|}{$\mathrm{SmScO}_{3}$} \\
\hline$a=0.3983 \mathrm{~nm}$ & $0.0078 \mathrm{~nm}(2.0 \%)$ & $-0.0022 \mathrm{~nm}(-0.5 \%)$ & 0.003 & $0.2 \mathrm{pm} / \mathrm{V}$ \\
\hline$b=0.3991 \mathrm{~nm}$ & $0.0086 \mathrm{~nm}(2.2 \%)$ & $-0.0014 \mathrm{~nm}(-0.3 \%)$ & - & - \\
\hline
\end{tabular}


In more complex configurations, additional complications can arise from lateral force cross-talk, ${ }^{36}$ cantilever flexure modes, ${ }^{37}$ and detection. ${ }^{38}$ These are discussed below.

The ferroelectric domain structures are predicted by the phase-field method coupled with microelasticity and electrostatics. ${ }^{39,40}$ In this approach, we use the components of polarization $P(x)=\left[P_{1}(\mathbf{x}), P_{2}(\mathbf{x}), P_{3}(\mathbf{x})\right]$, as order parameters, and their temporal evolution, thus the domain structure, is described by the time dependent Ginzburg-Landau (TDGL) equations,

$$
\frac{\partial P_{i}(\mathbf{x}, t)}{\partial t}=-L \frac{\delta F}{\delta P_{i}(\mathbf{x}, t)},(i=1,2,3),
$$

where $\mathbf{x}=\left(x_{1}, x_{2}, x_{3}\right)$ is the coordinate, $t$ is time, $L$ is the kinetic coefficient related to the ferroelectric domain wall mobility, and $F$ is the total free energy of the system, which is given by

$$
F=F_{\text {bulk }}\left(P_{i}\right)+F_{\text {elas }}\left(P_{i}, \varepsilon_{i j}\right)+F_{\text {elec }}\left(P_{i}, E_{i}\right)+F_{\text {grad }}\left(P_{i, j}\right),
$$

where $F_{\text {bulk }}, F_{\text {elas }}, F_{\text {elec }}$, and $F_{\text {grad }}$ are the corresponding bulk chemical, elastic, electrostatic, and gradient energies, respectively, $\varepsilon_{\mathrm{ij}}$ is the elastic strain, $E_{\mathrm{i}}$ is the electric field components induced from dipole-dipole interactions, and $P_{\mathrm{i}, \mathrm{j}}=\partial P_{\mathrm{i}} /$ $\partial x_{\mathrm{j}}$. The mathematical expressions and the numerical coefficients for obtaining the different energy contributions can be found in Refs. 41 and 42 . The cell grid matrix employed here is $\left(64 \Delta x_{1} * 64 \Delta x_{2}\right) * N \Delta x_{3}$, where the $\Delta x_{1}=\Delta x_{2}=1 \mathrm{~nm}$, $\Delta x_{3}=0.5 a_{S T}$ with $a_{S T}=0.3905 \mathrm{~nm}$, and $N=2(n+m)$ for a $\left(\mathrm{BaTiO}_{3}\right)_{n} /\left(\mathrm{SrTiO}_{3}\right)_{m}$ superlattice. We assume that the top surface of the superlattice and the film/substrate interface are charge-compensated along the $x_{3}$ direction and model the superlattice as a periodic structure.
In the simulation, the in-plane strains, $e_{11}$ and $e_{22}$, imposed on the superlattice are calculated from the lattice parameter difference

$$
e_{11}=e_{22}=\frac{a_{\text {sub }}-a_{\text {sup }}}{a_{\text {sup }}},
$$

where $\mathrm{a}_{\text {sub }}$ and $\mathrm{a}_{\text {sup }}$ are the lattice pseudo cubic lattice constants ${ }^{43}$ and superlattices, shown in Table I.

\section{RESULTS}

Figure 2 compares the amplitude images of $\left[\left(\mathrm{BaTiO}_{3}\right)_{n} /\right.$ $\left.\left(\mathrm{SrTiO}_{3}\right)_{m}\right]_{p}$ superlattices grown on the three substrates, and Fig. 3 shows the corresponding phase-field predictions of domain morphologies. The polarization directions of the colored domains are indicated by the corresponding arrows. All films were extremely flat, with topographic variations $<1 \mathrm{~nm}$ over micron-sized areas.

For the case of the $\left[\left(\mathrm{BaTiO}_{3}\right)_{3} /\left(\mathrm{SrTiO}_{3}\right)_{4}\right]_{25} p$ superlattice grown on $\mathrm{SrTiO}_{3}$, the $\mathrm{SrTiO}_{3}$ layers experience no strain while the $\mathrm{BaTiO}_{3}$ layer experiences a $2.5 \%$ compressive strain. Figures 2(a) and 2(d) shows that the morphology consists of meandering domains with sizes ranging from $20 \mathrm{~nm}$ to $50 \mathrm{~nm}$. (The circular structures are topographic artifacts). The theoretical prediction for the domain morphology is compared in Fig. 3(a) showing the $\mathrm{BaTiO}_{3}$ at the surface and Fig. 3(d) the $\mathrm{SrTiO}_{3}$ at the surface. In this case, the theory predicts that both the $\mathrm{SrTiO}_{3}$ and $\mathrm{BaTiO}_{3}$ are tetragonal with the long axis perpendicular to the substrate. The $\mathrm{SrTiO}_{3}$ layer is polarized and aligned in the direction of the $\mathrm{BaTiO}_{3}$ polarization resulting in relatively large $(30 \mathrm{~nm}-60 \mathrm{~nm}) c+$ and $c$-domains. The calculated volume average of the vertical
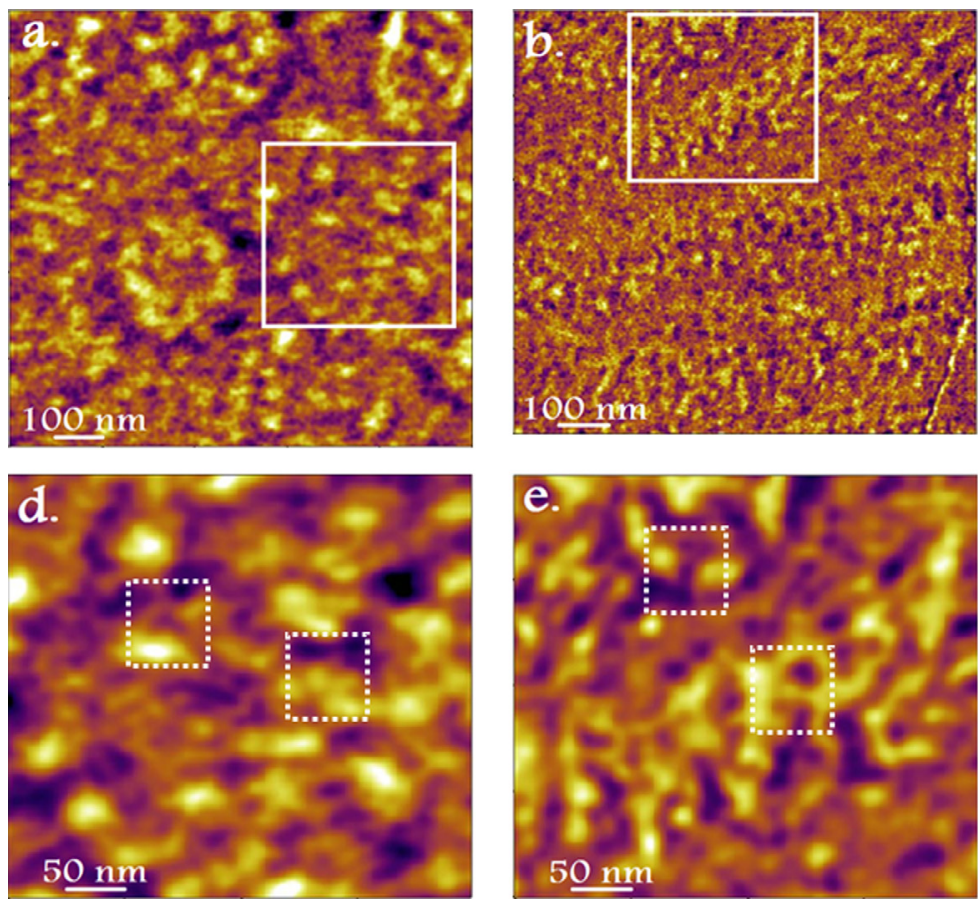
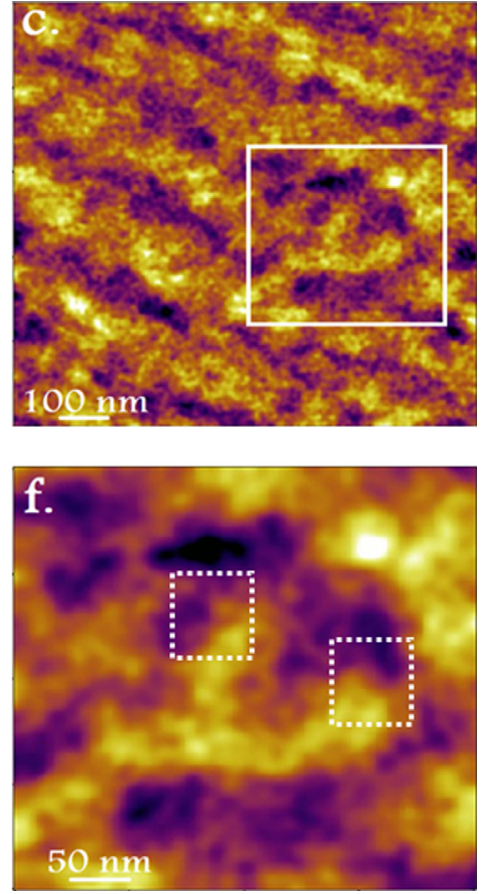

FIG. 2. Amplitude images of the $\left[\left(\mathrm{BaTiO}_{3}\right)_{n} /\left(\mathrm{SrTiO}_{3}\right)_{m}\right]_{p}$ superlattices grown on three substrates. (a) and (d) $=\mathrm{SrTiO}_{3}$, (b) and (e) $=\mathrm{GdScO}_{3}$, (c) and (f) $=\mathrm{SmScO}_{3}$. (a)-(c) are $1 \mu \mathrm{m}$ scan sizes. (d)-(f) are expanded regions designated by the box. The boxes in (d)-(f) are the size of the calculated images. Images were acquired with tip-free resonance $=79.14 \mathrm{kHz}, k=2.23 \mathrm{~N} / \mathrm{m}$, contact load $\sim 60 \mathrm{nN}$, and oscillation amplitude $=6.6 \mathrm{~V}$. 

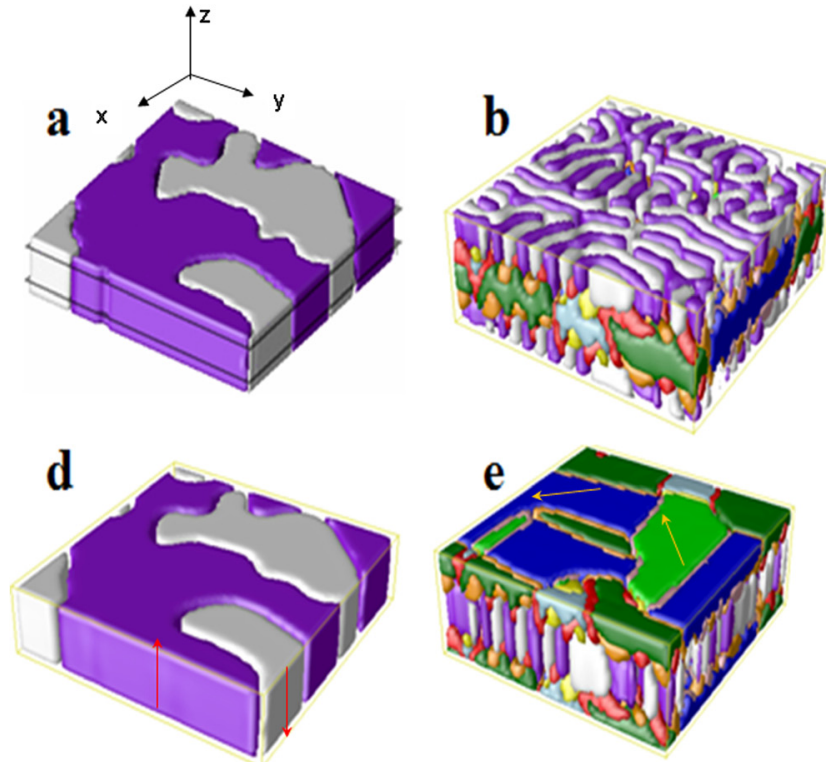

polarization is $|\mathrm{Pz}|_{\text {average }}=0.103 \mathrm{~cm}^{-2}$. The white squares in Fig. 2(d) outline areas of the same size as those included the calculations. The size and general shape of the domains agree exceptionally well with experiment.

Figures 2(b) and 2(e) and Figs. 3(b) and 3(e) compare the experimental and theoretical domain morphologies of the $\left[\left(\mathrm{BaTiO}_{3}\right)_{8} /\left(\mathrm{SrTiO}_{3}\right)_{4}\right]_{40}$ superlattices grown on $\mathrm{GdScO}_{3}$ in which the $\mathrm{SrTiO}_{3}$ layer would experience a $1.6 \%$ tensile strain, while the $\mathrm{BaTiO}_{3}$ layer experiences a small, $0.9 \%$ compressive strain. This sample has the lowest average strain of the three examined here. The calculations predict elongated $\mathrm{c}+$ and $\mathrm{c}$ - domains with $\sim 10 \mathrm{~nm}$ widths in the $\mathrm{BaTiO}_{3}$ layers. The $\mathrm{SrTiO}_{3}$ layers are predicted to develop in-plane orthorhombic domains with the size of $30-50 \mathrm{~nm}$. The calculated volume average of the vertical polarization is $|\mathrm{Pz}|_{\text {average }}$ $=0.0470 \mathrm{~cm}^{-2}$. The experimentally observed polarization morphology consists of elongated contrast variations with diameters on the order of $25 \mathrm{~nm}$ and lengths on the order of $100 \mathrm{~nm}$ with intermittent regions of intermediate amplitude. Note that vertical PFM probes a depth of tens of nanometers, i.e., through the entire superlattice, accessing only the vertical projection of polarization vector. The $\mathrm{c}+$, c- domains in Figs. 3(b) and 3(e) are of the same dimensions as the tip contact area, so most of the image contrast is due to the tip accessing and averaging over several domains. It is interesting to note that the variations in contrast have the same dimensions as induced polarization in the underlying $\mathrm{SrTiO}_{3}$ layer.

Figures 2(c) and 2(f) and Figs. 3(c) and 3(g) compare the experimental and theoretical domain morphologies of the $\left[\left(\mathrm{BaTiO}_{3}\right)_{8} /\left(\mathrm{SrTiO}_{3}\right)_{4}\right]_{40} p$ superlattice grown on $\mathrm{SmScO}_{3}$. In this case, the experimentally determined polarization contrast exhibits relatively large-scale periodic in-plane variation with dimensions of $\sim 160 \mathrm{~nm}$, which leads us to consider the potential impact of in-plane strain variations. The substrate in-plane lattice dimensions differ by $\sim 10 \%(0.0078 \mathrm{~nm}$ vs. $0.0086 \mathrm{~nm}$ ). This leads to a $2.0 \%$ tensile strain in one direction in the $\mathrm{SrTiO}_{3}$ layers and a $2.2 \%$ strain in the orthogonal direction. Similarly, $\mathrm{BaTiO}_{3}$ layers experience a very small compressive strain; $0.5 \%$ in one direction and $0.3 \%$ in the
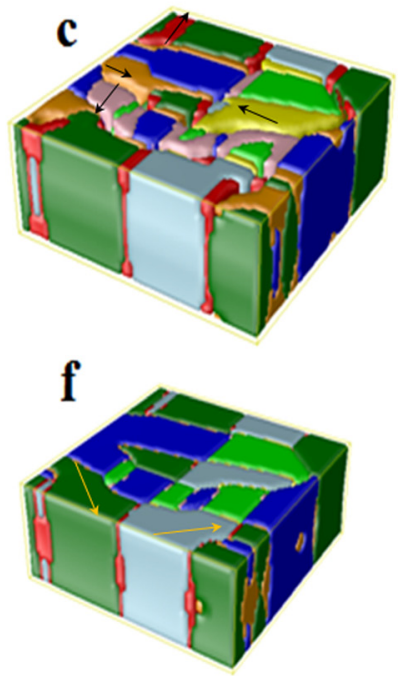

FIG. 3. Calculated ferroelectric domain images of the $\left[\left(\mathrm{BaTiO}_{3}\right)_{n} /\left(\mathrm{SrTiO}_{3}\right)_{m}\right]_{p}$ superlattices grown on three substrates. (a) and (d) on $\mathrm{SrTiO}_{3}$, (b) and (e) on $\mathrm{GdScO}_{3}$, (c) and (f) on $\mathrm{SmScO}_{3}$. (a)-(c) show domains with a $\mathrm{BaTiO}_{3}$ terminating layer, while (d)-(f) show domains with a $\mathrm{SrTiO}_{3}$ terminating surface. All calculations are of $64 \times 64 \mathrm{~nm}$ cells. The colors correspond to the domains with polarizations indicated the arrows.

other (see Table I). The theoretical prediction is for the energy minimization to be accomplished through in-plane rotations of the polarization in the $\mathrm{BaTiO}_{3}$ layer and in-plane polarization in the $\mathrm{SrTiO}_{3}$ layer, resulting in a combination of tetragonal and orthorhombic structures. The calculated volume average of the vertical polarization is $|\mathrm{Pz}|_{\text {average }}$ $=0.0 .003 \mathrm{~cm}^{-2}$. This domain configuration would result in almost zero perpendicular deformation; however, deformation contrast is observed in Figs. 2(c) and 2(f).

\section{DISCUSSION}

The first observation is that there are distinct differences in the measured polarization and domain morphologies between the films grown on substrates with varying degrees of strain. The calculations predict that both the $\mathrm{SrTiO}_{3}$ and $\mathrm{BaTiO}_{3}$ layers will be polarized and that the layer with the most strain will dictate the polarization direction. For example, in the sample grown on $\mathrm{SrTiO}_{3}$ the $\mathrm{BaTiO}_{3}$ is in compression and, therefore, is polarized "out of plane," i.e., perpendicular to the substrate. The $\mathrm{BaTiO}_{3}$ induces polarization in the $\mathrm{SrTiO}_{3}$ in the same direction, Fig. 3(a). For the case of the $\mathrm{SmScO}_{3}$ substrate where the $\mathrm{SrTiO}_{3}$ is in tension while the $\mathrm{BaTiO}_{3}$ compression is relatively small, the predicted polarization in both layers is "in plane," as dictated by the $\mathrm{SrTiO}_{3}$, Fig. 3(c). For the intermediate case, in which the magnitudes of the strains are similar, the $\mathrm{BaTiO}_{3}$ layer adopts an "out-of-plane" morphology reminiscent of the stripe domains in single component films, which is compensated by an "in-plane" morphology in the $\mathrm{SrTiO}_{3}$ layers. Given these predictions, it is unsurprising that the experimentally inferred polarization variation differs with strain. It is interesting that the character of the morphologies, e.g., the lateral size, shape, and correlations of the domains, is substantially and perhaps characteristically different.

To compare these predictions to experiment, note that the PFM measurements probe the piezoelectric response which, in the general case, is related to a tensor containing mechanical and electromechanical coupling properties. It is 
challenging to quantitatively extract properties from the PFM response. ${ }^{44}$ We have designed the samples and experiment to constrain several variables such that some simplifying assumption can be made. $\mathrm{BaTiO}_{3}$ and $\mathrm{SrTiO}_{3}$ are high symmetry crystals, the films are thin, and the orientation is constrained such that the (100) is perpendicular to the surface. For $\mathrm{c}+$ and $\mathrm{c}$ - domains, the majority of the response is related to the ferroelectric dipole. For more complicated domain configurations including lower symmetry phases, the piezoelectric response is related to polarization in a more complicated manner. In these cases, the morphologies are compared and trends in piezoelectric response identified.

A first comparison is the predicted volume averaged polarization and the average measured piezoresponse, Table I. The films grown on $\mathrm{SrTiO}_{3}$ should exhibit the largest vertical component of polarization, and the film on $\mathrm{SmScO}_{3}$ should exhibit the smallest component. We first note that there is qualitative agreement between the trends in the measurement and predicted polarization; both decrease as the strain induces a larger component of the polarization to the in-plane orientation. The experimentally measured decrease is not as large as that predicted. This is discussed in detail below.

The configuration of the film on the $\mathrm{SrTiO}_{3}$ substrate is ideal in that the orientation of the polarization is completely vertical so the probe directly accesses the polarization vector. As a consequence, both the volume averaged response and the domain morphology shown in Fig. 4(a) agree well with predictions. Note that a film consisting entirely of $\mathrm{c}+$ and c-domains should exhibit no variations in deformation amplitude, i.e., image contrast, except for tip artifacts at the domain boundaries. Ideally, the direction, positive or negative, of the polarization vector does not affect the amplitude of the deformation. We have shown previously ${ }^{23}$ that this polarization asymmetry is indicative of an internal electric field and can be used to determine the magnitude of the electric field. The experimentally observed asymmetry corresponds to a field of $7-9 \times 10^{6} \mathrm{~V} / \mathrm{m}$, perpendicular to the plane of the surface. The field is well below (10\% to $30 \%$ of $\mathrm{E}_{\mathrm{c}}$ ) the expected coercive field for epitaxial films. ${ }^{47-50} \mathrm{We}$ do not determine the origin of the internal field, but the difference between compensation at the top and bottom of the film would cause such a field.

The domain configuration in the film on the $\mathrm{SmScO}_{3}$ substrate is predicted to be entirely "in plane" and contribute negligible vertical contrast. Indeed the measured value is a factor of 4 smaller than the completely vertical case, but it is not zero. This could be a consequence of cross-talk between the lateral and vertical piezo response discussed by McGilly et $a l .{ }^{36}$ The presence of an internal electric field would, however, act to increase the amount vertical polarization and affect the domain morphology. With the complex domain configuration, in this case, it is not possible to estimate the internal field strength implied by the measurements, but its presence would account for the small, but measurable vertical polarization. Similarly, the presence of an internal electric field for the intermediate case accounts for the higher than expected ( $\sim 50 \%$ higher) measured average polarization.

Figure 4 compares the domain morphologies for the three samples on a size scale commensurate with the calculation. As noted above, for the case of vertically orientated domains, the agreement is excellent. With the volumeaveraged measurement, it is not possible to infer the level of detail shown in Fig. 3 on films that contain 3-D domain structure, but some interesting observations can be made. The measured domain morphology in the film on the $\mathrm{GdScO}_{3}$ substrate exhibits a threading worm-like morphology similar to the prediction for the $\mathrm{BaTiO}_{3}$ layer but on a larger length scale, as seen in Figs. 2(b) and 2(e). On a smaller scale, the domain size is between those predicted to be in the $\mathrm{SrTiO}_{3}$ and $\mathrm{BaTiO}_{3}$ layers, respectively (Fig. 4(b)). The small-scale morphology on the $\mathrm{SmScO}_{3}$ substrate exhibits domain size and shape similar to the prediction of the inplane domains, suggesting that the internal electric field affects the domains differently, which might be expected. Compare Figs. 3(c) and 4(c).

Finally, at the larger length scales shown in Fig. 2, the film on the $\mathrm{SmScO}_{3}$ substrate exhibits clear variations of contrast, which are most clearly evident in Fig. 2(c). There are line-like variations with periodicity of $\sim 160 \mathrm{~nm}$ and
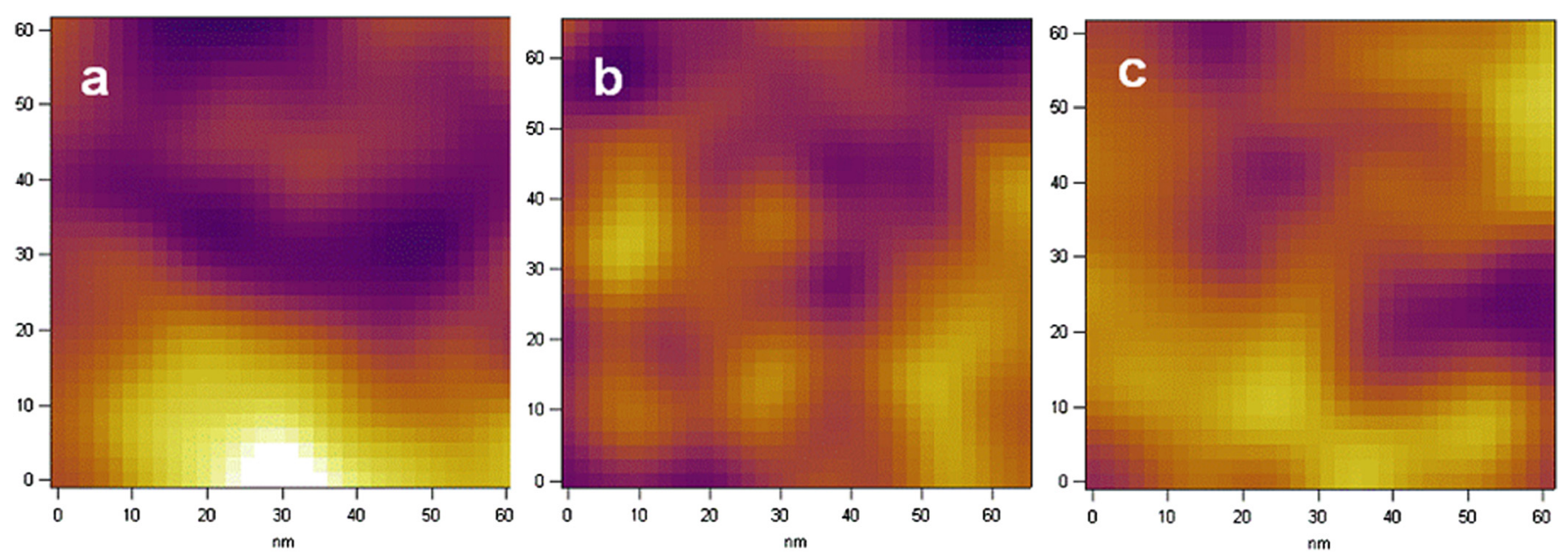

FIG. 4. Amplitude images of areas the same size as the calculations for the $\left[\left(\mathrm{BaTiO}_{3}\right)_{n} /\left(\mathrm{SrTiO}_{3}\right)_{m}\right]_{p}$ superlattices grown on (a) $\mathrm{SrTiO}_{3}$, (b) $\mathrm{GdScO}_{3}$, and (c) $\mathrm{SmScO}_{3}$. 
contrast variations along the line. Since the substrate inplane unit cell dimensions are not equal, $a=0.3983 \mathrm{~nm}$ and $\mathrm{b}=0.3991 \mathrm{~nm}$, it is interesting to consider the impact of this on domain structure. If we assume that the film is fully commensurate, or more specifically, that there is no periodic strain relief mechanism at the interface, then the strain in the film is different in orthogonal directions. The difference in the strain is small, $2.0 \%$ vs. $2.2 \%$ in the $\mathrm{SrTiO}_{3}$ layer and $-0.3 \%$ vs. $-0.5 \%$ in the $\mathrm{BaTiO}_{3}$ or $\sim 0.2 \%$. If this strain difference were accommodated by regions of vertical or more vertically oriented domains, the contrast in Fig. 2(c) implies $\sim 300$ unit cells in one state, 100 unit cells in another along the direction perpendicular to the line features. Much work needs to be done to examine this scenario, but if confirmed, it may serve as a strategy for fabricating patterned domains in ferroelectric films and superlattices.

\section{CONCLUSIONS}

A precise variant of PFM with quantitative consideration of point contact resonance variations was used to characterize the effect of strain on polarization in $\left[\left(\mathrm{BaTiO}_{3}\right)_{n} /\right.$ $\left.\left(\mathrm{SrTiO}_{3}\right)_{m}\right]_{p}$ superlattices. For the ideal case, in which polarization was perpendicular to the substrate, the measured polarization and domain morphology were in good agreement with phase-field calculations. This case allows the presence of an internal electric field in the thin film to be identified. The measured trend in polarization with strain state was in qualitative agreement with predictions, and the quantitative differences were consistent with the presence of internal electrical fields. Differences of domain morphology in heterostructured films with completely vertical and combined domain morphologies were predicted. Clear differences of domain morphology with lattice strain were observed. These were consistent with theoretical predictions if an internal field was invoked, but the possible effect of a lateral force contribution could not be excluded.

\section{ACKNOWLEDGMENTS}

The work at the University of Pennsylvania was partially supported by the National Science Foundation DMR \# 00-0805174. Facilities support by the Nano/Bio Interface Center is gratefully acknowledged. The work at Cornell was supported by the U.S. Department of Energy, Office of Basic Energy Sciences, Division of Materials Sciences and Engineering under Award \#DE-SCOO02334. The work at Penn State is supported by the Department of Energy Basic Sciences under the Grant No. DOE DE-FG02-07ER46417.

${ }^{1}$ N. A. Pertsev, A. G. Zembilgotov, and A. K. Tagantsev, Phys. Rev. Lett. 80, 1988-1991 (1998).

${ }^{2}$ E. D. Specht, H. M. Christen, D. P. Norton, and L. A. Boatner, Phys. Rev. Lett. 80, 4317-4320 (1998).

${ }^{3}$ M. Warusawithana, E. V. Colla, J. N. Eckstein, and M. B. Weissman, Phys. Rev. Lett. 90, 036802 (2003).

${ }^{4}$ M. Dawber, C. Lichtensteiger, M. Cantoni, M. Veithen, P. Ghosez, K. Johnston, K. M. Rabe, and J.-M. Triscone, Phys. Rev. Lett. 95, 177601 (2005).

${ }^{5}$ M. Dawber, K. M. Rabe, and J. F. Scott, Rev. Mod. Phys. 77, 1083-1130 (2005).
${ }^{6}$ H. N. Lee, H. M. Christen, M. F. Chisholm, C. M. Rouleau, and D. H. Lowndes, Nature (London) 433, 395 (2005).

${ }^{7}$ D. G. Schlom, L.-Q. Chen, C-B. Eom, K. M. Rabe, S. K. Streiffer, and J. M. Triscone, Annu. Rev. Mater. Res. 37, 589-626 (2007).

${ }^{8}$ E. Bousquet, M. Dawber, N. Stucki, C. Lichtensteiger, P. Hermet, S. Gariglio, J.-M. Triscone, and P. Ghosez, Nature (London) 452, 732 (2008).

${ }^{9}$ N. A. Pertsev, A. K. Tagantsev, and N. Setter, Phys. Rev. B 61, 825-829 (2000).

${ }^{10}$ J. H. Haeni, P. Irvin, W. Chang, R. Uecker, P. Reiche, Y. L. Li, S. Choudhury, W. Tian, M. E. Hawley, B. Craigo, A. K. Tagantsev, X. Q. Pan, S. K. Streiffer, L.-Q. Chen, S. W. Kirchoefer, J. Levy, and D. G. Schlom, Nature (London) 430, 758-761 (2004).

${ }^{11}$ E. Bousquet, N. Spaldin, and P. Ghosez, Phys. Rev. Lett. 104, 037601 (2010).

${ }^{12}$ M. Tyunina, J. Narkilahti, M. Plekh, R. Oja, R. M. Nieminen, A. Dejneka, and V. Trepakov, Phys. Rev. Lett. 104, 227601 (2010).

${ }^{13}$ C. J. Fennie and K. M. Rabe, Phys. Rev. Lett. 97, 267602 (2006).

${ }^{14}$ J. H. Lee and K. M. Rabe, Phys. Rev. Lett. 104, 207204 (2010).

${ }^{15}$ J. H. Lee, L. Fang, E. Vlahos, X. Ke, Y. W. Jung, L. Kourkoutis, J. W. Kim, P. Ryan, T. Heeg, M. Roeckerath, V. Goian, M. Bernhagen, R. Uecker, P. C. Hammel, K. M. Rabe, S. Kamba, J. Schubert, J. W. Freeland, D. A. Muller, C. J. Fennie, P. Schiffer, V. Gopalan, E. JohnstonHalperin, and D. G. Schlom, Nature (London) 466, 954-958 (2010).

${ }^{16}$ Z. Wu, N. Huang, Z. Liu, J. Wu, W. Duan, B.-L. Gu, and X.-W. Zhang, Phys. Rev. B 70, 104108 (2004)

${ }^{17}$ R. Takahashi, O. Dahl, E. Eberg, J. K. Grepstad, and T. J. Tybell, Appl. Phys. 104, 064109 (2008).

${ }^{18}$ S. K. Streiffer, J. A. Eastman, D. D. Fong, C. Thompson, A. Munkholm, M. V. Ramana Murty, O. Auciello, G. R. Bai, and G. B. Stephenson, Phys. Rev. Lett. 89, 067601 (2002).

${ }^{19}$ G. B. Stephenson and K. R. Elder, J. Appl. Phys. 100, 051601 (2006).

${ }^{20}$ S. Li, J. A. Eastman, J. M. Vetrone, R. E. Newnham, and L. E. Cross, Philos. Mag. B 76, 47-57 (1997).

${ }^{21}$ N. Sai, B. Meyer, and D. Vanderbilt, Phys. Rev. Lett. 84, 5636-5639 (2000).

${ }^{22}$ J. B. Neaton and K. M. Rabe, Appl. Phys. Lett. 82, 1586-1588 (2003).

${ }^{23}$ S. M. Nakhmanson, K. M. Rabe, and D. Vanderbilt, Phys. Rev. B 73, 060101 (2006).

${ }^{24}$ A. Soukiassian, W. Tian, D. A. Tenne, X. X. Xi, D. G. Schlom, N. D. Lanzillotti-Kimura, A. Bruchhausen, A. Fainstein, H. P. Sun, X. Q. Pan, A. Cros, and A. Cantarero, Appl. Phys. Lett. 90, 042909 (2007).

${ }^{25}$ S. M. Nakhmanson, K. M. Rabe, and D. Vanderbilt, Appl. Phys. Lett. 87, 102906 (2005).

${ }^{26}$ K. J. Choi, M. Biegalski, Y. L. Li, A. Sharan, J. Schubert, R. Uecker, P. Reiche, Y. B. Chen, X. Q. Pan, V. Gopalan, L.-Q. Chen, D. G. Schlom, and C. B. Eom, Science 306, 1005 (2004).

${ }^{27}$ D. A. Tenne, A. Bruchhausen, N. D. Lanzillotti-Kimura, A. Fainstein, R. S. Katiyar, A. Cantarero, A. Soukiassian, V. Vaithyanathan, J. H. Haeni, W. Tian, D. G. Schlom, K. J. Choi, D. M. Kim, C. B. Eom, H. P. Sun, X. Q. Pan, Y. L. Li, L. Q. Chen, Q. X. Jia, S. M. Nakhmanson, K. M. Rabe, and X. X. Xi, Science 313, 1614 (2006).

${ }^{28}$ S. V. Kalinin, A. N. Morozovska, L. Q. Chen, and B. J. Rodriguez, Rep. Prog. Phys. 73, 056502 (2010).

${ }^{29}$ K. Kathan-Galipeau, P. P. Wu, Y. L. Li, L. Q. Chen, A. Soukiassian, X. X. Xi, D. G. Schlom, and D. A. Bonnell, ACS Nano 5, 640-646 (2011).

${ }^{30}$ G. Koster, B. L. Kropman, G. J. H. M. Rijnders, and D. H. A. Blank, Appl. Phys. Lett. 73, 2920 (1998).

${ }^{31}$ A. Soukiassian, W. Tian, V. Vaithyanathan, J. H. Haeni, L.-Q. Chen, X. X. Xi, D. G. Schlom, D. A. Tenne, H. P. Sun, X. Q. Pan, K. J. Choi, C. B. Eom, Q. X. Jia, C. Constantin, R. M. Feenstra, M. Bernhagen, P. Reiche, and R. Uecker, J. Mater. Res. 23, 1417 (2008).

${ }^{32}$ B. J. Rodriguez, C. Callahan, and S. V. Kalinin, Nanotechnology 18, 475504 (2007).

${ }^{33}$ A. Gannepalli, D. Yablon, A. H. Tsou, and R. Proksch, Nanotechnology 22, 355705 (2011).

${ }^{34}$ S. Kalinin and D. A. Bonnell, Phys. Rev. B 64, 125408 (2002).

${ }^{35}$ S. Kalinin and D. A. Bonnell, J. Mater. Res. 17, 936 (2002).

${ }^{36}$ L. McGilly, D. Bryne, C. Harnagea, A. Shilling, and J. M. Gregg, J. Mater. Sci. 44, 5197 (2009).

${ }^{37}$ S. Kalinin, B. Rodriguez, S. Jesse, J. Shin, A. Baddorf, P. Gupta, J. Jaim, A. Gruverman, and D. B. Williams, Microsc. Microanal. 12, 206 (2006).

${ }^{38}$ B. D. Huey, C. Ramanujan, M. Bobji, J. Blendell, G. White, G. Szoszkiewicz, and A. Kulik, J. Electroceram. 12, 287 (2004).

${ }^{39}$ Y. L. Li, S. Y. Hu, Z. K. Liu, and L. Q. Chen, Appl. Phys. Lett. 78, 3878 (2001). 
${ }^{40}$ Y. L. Li, S. Y. Hu, Z. K. Liu, and L. Q. Chen, Acta Mater. 50, 395 (2002).

${ }^{41}$ Y. L. Li, L. E. Cross, and L. Q. Chen, J. Appl. Phys. 98, 064101 (2005).

${ }^{42}$ Y. L. Li, S. Y. Hu, D. Tenne, A. Soukiassian, D. G. Schlom, X. X. Xi, K. J. Choi, C. B. Eom, A. Saxena, T. Lookman, Q. X. Jia, and L. Q. Chen, Appl. Phys. Lett. 91, 112914 (2007).

${ }^{43}$ R. Uecker, B. Velickov, D. Klimm, R. Bertram, M. Bernhagen, K. M. Rabe, M. Albrecht, R. Fornari, and D. G. Schlom, J. Cryst. Growth 310, 2649-2658 (2008).

${ }^{44}$ S. Kalinin, B. Rodriguez, S. Jesse, E. Karapetian, B. Mirman, E. Eliseev, and A. Morozovska, Annu. Rev. Mat. Res. 37, 180 (2007).
${ }^{45}$ C. S. Ganpule, V. Nagarajan, H. Li, A. Ogale, D. Steinhauer, S. Aggarwal, E. Williams, R. Ramexh, and P. De Wolf, Appl. Phys. Lett. 77, 292 (2000).

${ }^{46}$ A. Agronin, M. Molotskii, Y. Rosenwaks, E. Strassburg, A. Boag, S. Mutchnik, and G. Rosenman, J. Appl. Phys. 97, 084312 (2005).

${ }^{47}$ S. M. Zanetti, P. R. Buero, E. Leite, E. Longo, and J. A. Varela, J. Appl. Phys. 89, 3416-3419 (2001).

${ }^{48}$ S. Choudhury, Y. L. Li, L. Q. Chen, and Q. X. Jia, Appl. Phys. Lett. 92, 142907 (2008).

${ }^{49}$ J. Y. Jo, Y. S. Kim, T. W. Noh, and J.-G. Yoon, Appl. Phys. Lett. 89, 232909 (2006).

${ }^{50}$ K. K. S. Abe, N. Yanase, and T. Kawakubo, Jpn. J. Appl. Phys., Part 1 36, 5846-5853 (1997). 